\title{
Spoke Model for Calculating Reliability Index and Safety Factor of Slopes
}

\author{
Ping Wang, ${ }^{1,2}$ Dongyan Liu, ${ }^{2}$ Haibin Huang, ${ }^{1}$ and Dongsheng Liu ${ }^{3}$ \\ ${ }^{1}$ Department of Military Civil Engineering, Logistical Engineering University of PLA, Chongqing 401331, China \\ ${ }^{2}$ College of Civil Engineering, Chongqing University, Chongqing 400045, China \\ ${ }^{3}$ Geological Prospecting Bureau of Chongqing, Chongqing 401121, China
}

Correspondence should be addressed to Dongyan Liu; cqudyliu@yahoo.com.cn

Received 4 December 2012; Revised 7 March 2013; Accepted 14 March 2013

Academic Editor: Panos Liatsis

Copyright (C) 2013 Ping Wang et al. This is an open access article distributed under the Creative Commons Attribution License, which permits unrestricted use, distribution, and reproduction in any medium, provided the original work is properly cited.

\begin{abstract}
Considering the disadvantages of the slice method commonly employed in reliability analysis of slopes, a novel method (Spoke model) was proposed for reliability analysis and safety factor calculation of slopes in this work based on geometrical relationship among slices. The safety factor and the coefficients of limit state function of slopes could be achieved with the Gaussian integral method. The minimum safety factor and the minimum reliability index, as well as their corresponding coordinates on the slip surface, can be calculated with the improved JC method and the searching method. A novel and practical method for reliability analysis of slopes has been achieved. With this method the slice process could be avoided, which helps to eliminate some calculation errors caused by oversimplified assumption. Moreover, the explicit expression of safety factor in this method requires no repeated iterative solution, which is employed in traditional slice methods, as well as can be developed into a limit state function required by calculation of the reliability index. It is demonstrated that this method works efficiently and succinctly in evaluation of reliability index and safety factor for soil slopes.
\end{abstract}

\section{Introduction}

Each slope (including stable and unstable slopes) possesses an inherent tendency to degrade to a more stable form and eventually make the slope tend to horizontal. Mass movement (slope rupture, landslide, etc.) may take place as a result of the shear failure. The Spoke model proposed in this work is mainly focused on the stability analysis and reliability analysis of simple slopes, where the concept of simple slope discussed in this work is of the slope morphology as shown in Figure 1, and the concept includes the following aspects: (1) the soil is homogeneous, and considering its geotechnical parameters it only includes weight density " $w$ ", cohesion " $c$ ", and internal friction angle " $\varphi$ "; (2) the underground water are not taken into consideration.

\section{Literature Review}

There are many methods to determine a slope slip surface. The center coordinate and the radius of a sliding circle were employed to determine the slip surface in one method $[1,2]$, while the center coordinate and the height of a sliding circle were introduced in another method [3]. A variety of methods have been applied to analyze the reliability of slopes. Based on traditional slice methods (such as Bishop method [3], Spencer model, Sarma model, and Janbu model $[4,5])$, the reliability index can be achieved with the calculated safety factor $F$ and the limit state function $Z=F-1$. In other cases the minimum reliability index was obtained with the limit state function on the slip surface where the safety factor reaches its minimum value [6]. These approaches are approximate methods for calculation of the reliability index, because all the expressions of safety factor in the slide methods are implicit, which require complicated iterative process when solving. Moreover, the slip surface corresponding to the minimum reliability index may not be the slip surface corresponding to the minimum safety factor [7].

The parallel slide method was employed to analyze the stability of slopes in Bishop method. The safety factor $F_{s}$ is 


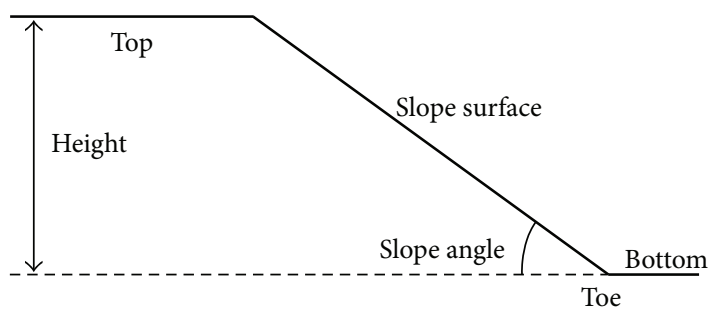

FIGURE 1: Illustration of a simple slope.

expressed as follows:

$$
F_{s}=\frac{\sum\left(1 / m_{a i}\right)\left[c_{i} b_{i}+\left(W_{i}-u_{i} b_{i}\right) \tan \varphi_{i}\right]}{\sum W_{i} \sin \alpha_{i}} .
$$

Obviously, the equation is implicit where the parameter $m_{a}$ contains $F_{s}$ as well; therefore, it can be solved only with the iterative method. Since the $F_{s}$ expression is implicit, the corresponding limit state function cannot be achieved when analyzing the stability of slopes with traditional slice methods.

\section{Spoke Model}

To achieve the limit state equation, a novel slide model (the Spoke model) is proposed in this work. With this model we can get the limit state equation from which the reliability index of slopes can be obtained.

Assuming that a slope is homogeneous, the height, slope angle, slope weight, angle of internal friction, and cohesion are $H_{0}, \alpha_{0}, w, \varphi$, and $c$, respectively. The slip surface is supposed to be a circular arc surface (Figure 2). Point $O$ is the center of the slope line. Points $O, M$, and $N$ are on the same line. The angle between line $N M$ and the slope line is $\psi$. Since $O O^{\prime} \perp M N$ and $O O^{\prime}=h$, the arc $N M$ is the slip line. Translate left the center $O^{\prime}$, the arc $N M$, segments, and intersections with a distance of $l$. The coordinates $(\psi, l, h)$ correspond to all possible slip surfaces, respectively.

The slip soil can be divided into three parts $S_{1}, S_{2}$, and $S_{3}$. Taking the second part $S_{2}$, for example, the center angle $\Delta \theta_{i}$ has crossing points AFDG with the slip line and slope line. The area of the $i$ th AFDG is $\Delta S_{2}=S_{\text {AFDG }}$. The radius of slip circle is $R_{0}$ which can be expressed in

$$
R_{0}=\left\{h^{2}+\left[\frac{H_{0}}{2 \sin \left(\alpha_{0}-\psi\right)}\right]^{2}\right\}^{1 / 2} .
$$

Considering $\Delta \theta_{i}$ is a small value,

$$
\begin{aligned}
S_{\mathrm{AFDG}} & =\left(R_{0}^{2}-{\overline{O^{\prime} D}}^{2}\right) \frac{\Delta \theta_{i}}{2}, \\
\overline{O^{\prime} D} & =\overline{O^{\prime} B}-\overline{B C}-\overline{C D} .
\end{aligned}
$$

Assume that

$$
\begin{gathered}
r=\angle O O^{\prime} C=\theta-\left(\alpha_{0}-\psi\right)=\theta+\psi-\alpha_{0}, \\
\overline{O^{\prime} B}=\frac{h}{\cos r}=\frac{h}{\cos \left(\theta+\psi-\alpha_{0}\right)} .
\end{gathered}
$$

In triangle $\triangle O B C$, according to Sine theorem, it can be achieved that

$$
\frac{\overline{B C}}{\sin \psi}=\frac{h \tan r}{\sin \left(r+90^{\circ}-\psi\right)}=\frac{h \tan r}{\cos (\psi-r)} .
$$

Combining (5) and (7),

$$
\overline{B C}=\frac{h \tan \left(\theta+\psi-\alpha_{0}\right) \sin \psi}{\cos \left(\theta-\alpha_{0}\right)} .
$$

Similarly, in triangle $\triangle O B C$,

$$
\overline{C D}=\frac{\overline{C P}}{\cos \left(\theta-\alpha_{0}\right)} \frac{l \sin \alpha_{0}}{\cos \left(\theta-\alpha_{0}\right)} .
$$

Combining (6), (8), (9), and (4),

$$
\begin{aligned}
\overline{O^{\prime} D}= & \frac{h}{\cos \left(\theta+\psi-\alpha_{0}\right)}-\frac{h \tan \left(\theta+\psi-\alpha_{0}\right) \sin \psi}{\cos \left(\theta-\alpha_{0}\right)} \\
& -\frac{l \sin \alpha_{0}}{\cos \left(\theta-\alpha_{0}\right)} .
\end{aligned}
$$

Combining (3) and (10),

$$
\begin{aligned}
& S_{\text {AFDG }} \\
& =\frac{\Delta \theta_{i}}{2}\left\{R_{0}^{2}-\left[\frac{h}{\cos \left(\theta+\psi-\alpha_{0}\right)}-\frac{h \tan \left(\theta+\psi-\alpha_{0}\right) \sin \psi}{\cos \left(\theta-\alpha_{0}\right)}\right.\right. \\
& \left.\left.-\frac{l \sin \alpha_{0}}{\cos \left(\theta-\alpha_{0}\right)}\right]^{2}\right\}
\end{aligned}
$$

Assuming that the area of the $i$ th slice is $S_{i}$, bulk density and weight of the soil are $w_{i}$ and $W_{i}$, respectively, as follows:

$$
W_{i}=w_{i} S_{i} .
$$

In the second part, $S_{i}$ is determined by (11).

$$
S_{i}=S_{\mathrm{AFDG}} \cdot
$$

Similarly, the $S_{i}$ expression of the first and third parts can be achieved, respectively, as follows:

$$
\begin{aligned}
& S_{i}=\frac{\Delta \theta_{i}}{2}\left\{R_{0}^{2}-\frac{R_{0} \sin \left[\arcsin (h / R)+\alpha_{0}-\psi\right]}{\cos \theta}\right\}, \\
& S_{i}=\frac{\Delta \theta_{i}}{2}\left\{R_{0}^{2}-\frac{R_{0} \cos \left[\arccos (h / R)+\alpha_{0}-\psi\right]}{\cos \theta}\right\} .
\end{aligned}
$$

Shear forces $X_{i}$ and $X_{i+1}$ pass through center $O^{\prime}$, perpendicular to the slip surface. The sideward normal forces are $E_{i}$ and $E_{i+1}$, respectively. According to the balance condition along the direction of each radial line,

$$
\begin{aligned}
W_{i} \cos \theta_{i} & +X_{i+1} \cos \left(\frac{\Delta \theta_{i}}{2}\right)-X_{i} \cos \left(\frac{\Delta \theta_{i}}{2}\right) \\
& +E_{i} \sin \left(\frac{\Delta \theta_{i}}{2}\right)+E_{i+1} \sin \left(\frac{\Delta \theta_{i}}{2}\right)-N_{i}=0
\end{aligned}
$$




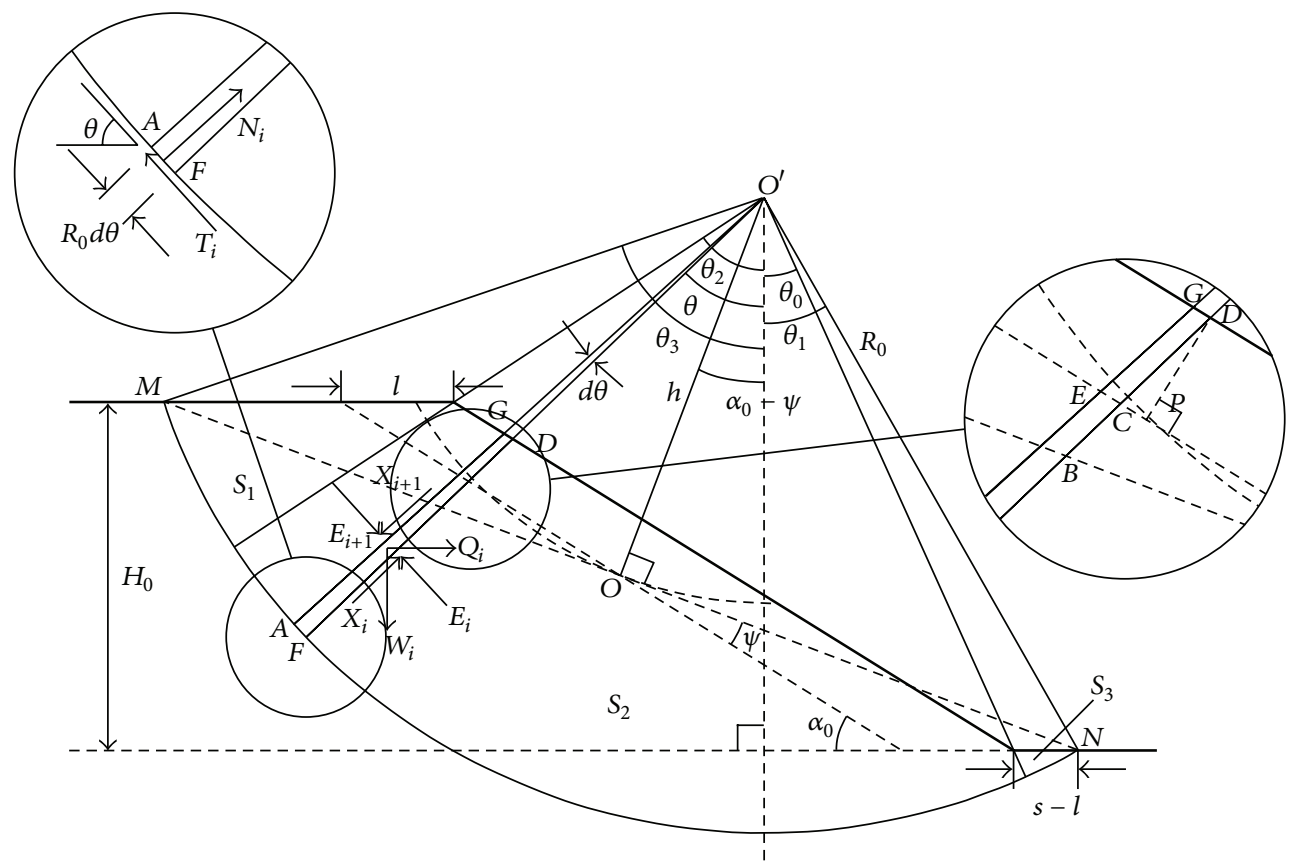

FIGURE 2: Spoke model.

Since $\Delta \theta_{i}$ is a definitely small value,

$$
\begin{gathered}
\cos \left(\frac{\Delta \theta_{i}}{2}\right)=1 \\
E_{i} \sin \left(\frac{\Delta \theta_{i}}{2}\right)+E_{i+1} \sin \left(\frac{\Delta \theta_{i}}{2}\right) \\
=\frac{E_{i} \Delta \theta_{i}}{2}+\frac{\left(E_{i}+\Delta E_{i}\right) \Delta \theta_{i}}{2}=E_{i} \Delta \theta_{i} .
\end{gathered}
$$

Combining (16), (17), and (18),

$$
W_{i} \cos \theta_{i}+X_{i+1}-X_{i}+E_{i} \Delta \theta_{i}-N_{i}=0 \text {. }
$$

In the limit equilibrium, the sum of all the force moments on the center should be zero; therefore,

$$
\sum W_{i} R_{i} \sin \theta_{i}-\sum T_{i} R_{0}+\sum Q_{i} e_{i}=0
$$

where $Q_{i}$ and $e_{i}$ are the inertia force of earthquake and its tension arm, respectively. $R_{i}$ is the distance between center of the circle and the weight center of a slice. The shear stress at the bottom of the slice can be expressed as

$$
T_{i}=\tau l_{i}=\frac{\tau_{f}}{F_{s}} l_{i}=\frac{c_{i} l_{i}}{F_{s}}+\left(N_{i}-u_{i} l_{i}\right) \frac{\tan \varphi_{i}}{F_{s}} .
$$

Combining (19), (20) and (21), the safety factor $F_{s}$ can be achieved.

$F_{s}=\frac{R_{0} \sum\left[c_{i} l_{i}+\left(W_{i} \cos \theta_{i}+X_{i+1}-X_{i}+E_{i} \Delta \theta_{i}-u_{i} l_{i}\right) \tan \varphi_{i}\right]}{\sum W_{i} R_{i} \sin \theta_{i}+\sum Q_{i} e_{i}}$, where the sum of all the force moments of the slide friction forces on the center of the circle resulting from shear stresses should be zero. Therefore,

$$
\begin{gathered}
R_{0} \tan \varphi \sum X_{i}=0, \\
l_{i}=R_{0} \Delta \theta_{i} \\
F_{s}=\frac{R_{0} \sum\left[c R_{0} \Delta \theta_{i}+\left(W_{i} \cos \theta_{i}+E_{i} \Delta \theta_{i}-u_{i} R_{0} \Delta \theta_{i}\right) \tan \varphi\right]}{\sum W_{i} R_{i} \sin \theta_{i}+\sum Q_{i} e_{i}} .
\end{gathered}
$$

Compared with the $F_{s}$ expression in Bishop method, (23) possesses three obvious advantages as follows: (i) the shear stress between the soil strips is not involved in (23); (ii) it is an absolute expression without $F_{s}$ in its right part, which requires no repeated iterative solution; (iii) (23) has a clear physical meaning. $F_{s}$ is the ratio of the total resisting moment to the total sliding moment.

When the slope is sliced infinitely, as well as the interstitial pressure and the seismic inertial force are not taken into consideration, the safety factor can be expressed in the integration form. Combining (13), (16), and (23),

$$
F_{s}=\frac{\left(c R_{0}^{2} / w\right) \int_{\theta_{0}}^{\theta_{3}} d \theta+R_{0} \tan \varphi \times T}{\int_{\theta_{0}}^{\theta_{1}} S_{1} R_{1} \sin \theta d \theta+\int_{\theta_{1}}^{\theta_{2}} S_{2} R_{2} \sin \theta d \theta+\int_{\theta_{2}}^{\theta_{3}} S_{3} R_{3} \sin \theta d \theta},
$$

where $T=\int_{\theta_{0}}^{\theta_{1}}\left[S_{1} \cos \theta+E_{1}(\theta)\right] d \theta+\int_{\theta_{1}}^{\theta_{2}}\left[S_{2} \cos \theta+E_{2}(\theta)\right] d \theta+$ $\int_{\theta_{2}}^{\theta_{3}}\left[S_{3} \cos \theta+E_{3}(\theta)\right] d \theta$. 
Therefore,

$$
\begin{aligned}
& f=\frac{c R_{0}^{2}}{w} \int_{\theta_{0}}^{\theta_{3}} d \theta+R_{0} \tan \varphi \\
& \quad \times\left\{\int_{\theta_{0}}^{\theta_{1}}\left[S_{1} \cos \theta+E_{1}(\theta)\right] d \theta\right. \\
& \left.\quad+\int_{\theta_{1}}^{\theta_{2}}\left[S_{2} \cos \theta+E_{2}(\theta)\right] d \theta+\int_{\theta_{2}}^{\theta_{3}}\left[S_{3} \cos \theta+E_{3}(\theta)\right] d \theta\right\} \\
& \quad \div\left(\int_{\theta_{0}}^{\theta_{1}} S_{1} R_{1} \sin \theta d \theta\right. \\
& \left.\quad+\int_{\theta_{1}}^{\theta_{2}} S_{2} R_{2} \sin \theta d \theta+\int_{\theta_{2}}^{\theta_{3}} S_{3} R_{3} \sin \theta d \theta\right),
\end{aligned}
$$

where $S_{j} d \theta(j=1,2,3)$ in (24) and (25) is the integrated area element, $R_{j}(j=1,2,3)$ is the distance between the slice center and the center of the circle, and $S_{j} R_{j} d \theta(j=1,2,3)$ represents the quadrature. Combining (11)-(15), they could be expressed as the functions of the parameters $H_{0}, \alpha_{0}, \psi, l$, and $h$ as follows:

$$
\begin{array}{r}
S_{j} d \theta=\left(R_{0}^{2}-r_{j}^{2}\right) \frac{d \theta}{2}, \\
R_{j}=\frac{2\left(R_{0}^{2}+R_{0} r_{j}+r_{j}^{2}\right)}{3\left(R_{0}+r_{j}\right)}, \\
S_{j} R_{j} d \theta=\left(R_{0}^{3}-r_{j}^{3}\right) \frac{d \theta}{3}, \\
(j=1,2,3),
\end{array}
$$

where

$$
\begin{gathered}
r_{1}=\frac{R_{0} \sin \left[\arcsin \left(h / R_{0}\right)+\alpha_{0}-\psi\right]}{\cos \theta}, \\
r_{2}=\frac{h}{\cos \left(\theta+\psi-\alpha_{0}\right)} \\
-\frac{h \tan \left(\theta+\psi-\alpha_{0}\right) \sin \psi}{\cos \left(\theta-\alpha_{0}\right)}-\frac{l \sin \alpha_{0}}{\cos \left(\theta-\alpha_{0}\right)}, \\
r_{3}=\frac{R_{0} \cos \left[\arccos \left(h / R_{0}\right)+\alpha_{0}-\psi\right]}{\cos \theta} .
\end{gathered}
$$

In (24) and (25), the upper/lower limit of integral $\theta_{i}(i=$ $0,1,2,3)$ represents the central angle between the line $O^{\prime} H$ and another segment whose two endpoints are the center of circle and the endpoint of the slide slope segment in the circle arc, which can be expressed as the functions of the parameters $H_{0}, \alpha_{0}, \psi, l$, and $h$ as follows:

$$
\begin{gathered}
\theta_{0}=-\arccos \left(\frac{h}{R_{0}}\right)+\alpha_{0}-\psi, \\
\theta_{1}=-\arcsin \frac{R_{0} \cos \left[\arcsin \left(h / R_{0}\right)+\alpha_{0}-\psi\right]-(s-l)}{\left\{R_{0}^{2}+(s-l)^{2}-2 R_{0}(s-l) \cos \left[\arcsin \left(h / R_{0}\right)+\alpha_{0}-\psi\right]\right\}^{1 / 2}}, \\
\theta_{2}=\arcsin \frac{R_{0} \sin \left[\arccos \left(h / R_{0}\right)+\alpha_{0}-\psi\right]-(s+l)}{\left\{R_{0}^{2}+(s+l)^{2}-2 R_{0}(s+l) \cos \left[\arcsin \left(h / R_{0}\right)-\alpha_{0}+\psi\right]\right\}^{1 / 2}}, \\
\theta_{3}=\arccos \left(\frac{h}{R_{0}}\right)+\alpha_{0}-\psi .
\end{gathered}
$$

In (26), (27), and (28),

$$
\begin{gathered}
R_{0}=\left\{h^{2}+\left[\frac{H_{0}}{2 \sin \left(\alpha_{0}-\psi\right)}\right]^{2}\right\}^{1 / 2}, \\
s=\frac{H_{0} \sin \psi}{2 \sin \left(\alpha_{0}-\psi\right) \sin \alpha_{0}} .
\end{gathered}
$$

In (24) and (25), $E(\theta)$ can be achieved by solving differential equations. The differential equation in the tangential direction of the $i$ th slice slope can be expressed as follows:

$$
\Delta E_{i}=-W_{i} \sin \theta+N_{i} \tan \varphi .
$$

Combining (20) and (31),

$$
\Delta E_{i}=-W_{i} \sin \theta_{i}+\left(W_{i} \cos \theta_{i}+E_{i} \Delta \theta_{i}\right) \tan \varphi+\Delta X_{i} \tan \varphi .
$$

To simplify the calculation, let $\Delta X_{i}=\Delta E_{i} \tan \varphi, \mu=$ $\tan \varphi$, and (32) can be developed into a differential equation:

$$
d E(\theta)-\frac{\mu}{1-\mu^{2}} E(\theta) d \theta=w(\mu \cos \theta-\sin \theta) f(\theta) d \theta,
$$

where $f(\theta) d \theta$ is the area element, which can be expressed as follows with (26):

$$
f(\theta) d \theta=S_{j} d \theta=\left(R_{0}^{2}-r_{j}^{2}\right) \frac{d \theta}{2} .
$$

Let $\alpha=\mu /\left(1-\mu^{2}\right)$. The general solution of the linear differential (33) is

$$
E(\theta)=e^{\alpha \theta}\left[\int w(\mu \cos \theta-\sin \theta) f(\theta) e^{-\alpha \theta} d \theta+C\right],
$$

where $C$ is an arbitrary constant. Considering the complexity of $f(\theta)$, it is definitely complicated to solve the indefinite integral of the transcendental function. To simplify the calculation, $f(\theta)$ is replaced with a proper constant $M$. Within the interval $\theta>0$ in (26), $r_{j}$ is replaced with $\overline{{O O^{\prime}}^{\prime}}$. Equation (32) can be developed into

$$
E(\theta)=e^{\alpha \theta} M\left[\int w(\mu \cos \theta-\sin \theta) e^{-\alpha \theta} d \theta+C\right] .
$$




$$
\begin{aligned}
& \text { With } \\
& \int e^{-\alpha \theta} \cos \theta d \theta=\frac{1}{1+\alpha^{2}} e^{-\alpha \theta}(-\alpha \cos \theta+\sin \theta)+C_{1}, \\
& \int e^{-\alpha \theta} \sin \theta d \theta=\frac{1}{1+\alpha^{2}} e^{-\alpha \theta}(-\alpha \sin \theta-\cos \theta)+C_{2},
\end{aligned}
$$

(36) can be developed into

$$
\begin{aligned}
E(\theta)= & \frac{w M}{1+\alpha^{2}} \\
& \times\left\{[(1-\alpha \mu) \cos \theta+(\alpha+\mu) \sin \theta]+C_{3} e^{-\alpha \theta}+C_{4}\right\} .
\end{aligned}
$$

Assuming $E(\theta)=0$ when $\theta=\theta_{3}$,

$$
\begin{aligned}
E(\theta)=\frac{w M}{1+\alpha^{2}}\{[ & (1-\alpha \mu) \cos \theta+(\alpha+\mu) \sin \theta] \\
& \left.-e^{\alpha\left(\theta-\theta_{3}\right)}\left[(1-\alpha \mu) \cos \theta_{3}+(\alpha+\mu) \sin \theta_{3}\right]\right\} .
\end{aligned}
$$

When $\partial E(\theta) / \partial \theta=0$, we can obtain the maximum value of broadside normal stress $E_{m}(\theta)$ and its corresponding angle $\theta=\theta_{m}$. The normal stress would reduce to zero gradually from the cut-off point toward both directions. It can be analyzed that $\theta_{m}$ is in the interval $\theta>0$. Combining $E=$ $E\left(\theta_{m}\right) / 2$ and (24), (25), the safety factor can be calculated, and the limit state function can be determined.

Subsequently, combine (24)-(30). As for a certain slope (i.e., $\alpha_{0}$ and $H_{0}$ are fixed.), each group of $(\psi, l, h)$ would result in its corresponding safety factor and limit state function. Namely, safety factor and limit state function can be expressed as a function containing variables $\psi, l$, and $h$, respectively as follows:

$$
\begin{gathered}
F_{s}=g_{1}(\psi, l, h), \\
f=g_{2}(\psi, l, h, c, \varphi, w) .
\end{gathered}
$$

Considering the random variability of soil parameters, we could obtain the reliability index of each group of $(\psi, l, h)$. By varying the numerical values of $(\psi, l, h)$, the minimum values of reliability index and safety factor can both be obtained. The calculation of structure reliability index has been discussed in many reported works [9-13]. In a typical failure mode of a slope (Figure 3), the slip surface passes through both the top plane and bottom plane. Based on geometrical condition and the constraint relationship among $\psi, l$, and $h$, inequalities (41) can be obtained theoretically as follows:

$$
\begin{gathered}
0 \leq \psi<\alpha_{0}, \\
|l| \leq \frac{H_{0} \sin \psi}{2 \sin \alpha_{0} \sin \left(\alpha_{0}-\psi\right)}, \\
\frac{H_{0}}{2 \cos \left(\alpha_{0}-\psi\right)} \leq h \\
\leq \frac{\left(H_{0} / \sin \alpha_{0}\right)-\left((s-l) / \cos \left(\alpha_{0}-\psi\right)\right)}{2 \tan \left(\alpha_{0}-\psi\right)} .
\end{gathered}
$$

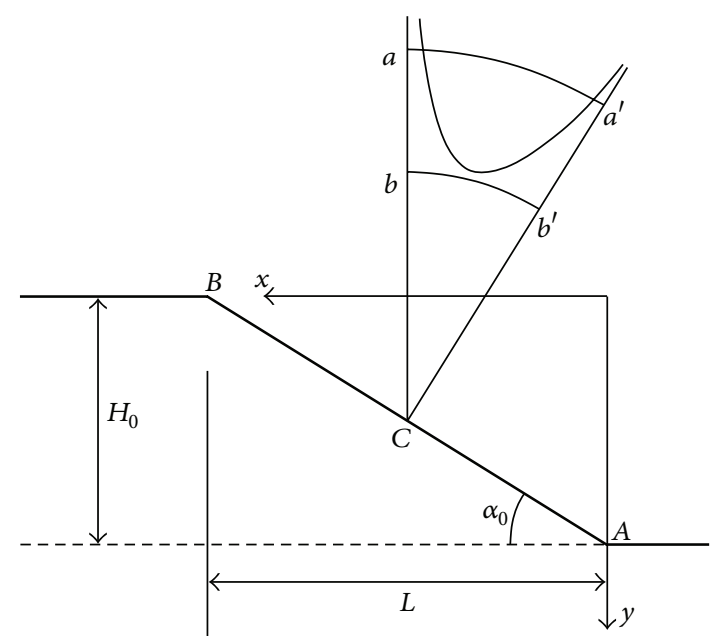

FIGURE 3: Area of most possible slip surface.

TAble 1: Calculation Results for Example (II).

\begin{tabular}{lcccc}
\hline & & \multicolumn{3}{c}{ Corresponding coordinate } \\
& & $x(\mathrm{~m})$ & $y(\mathrm{~m})$ & $r(\mathrm{~m})$ \\
\hline $\begin{array}{l}\text { Result achieved in } \\
{[8]}\end{array}$ & $K_{s \text { min }}=1.28$ & 59.21 & -84.72 & 152.13 \\
\hline & & \multicolumn{4}{c}{ Corresponding coordinate } \\
& & $\psi\left({ }^{\circ}\right)$ & $l(\mathrm{~m})$ & $h(\mathrm{~m})$ \\
\hline Result in this work & $K_{s \text { min }}=1.31$ & 2.18 & 6.35 & 115.22 \\
\hline
\end{tabular}

Pan Jia-zheng has stated that the center of critical slip surface is in the area formed by two circulars with radius of $H_{0} / 2 \tan \alpha_{0}$ and $3 H_{0} / 4 \tan \alpha_{0}$, respectively, central normal line and perpendicular bisector of the slope surface (Figure 3 ) [14]. By varying the values of $\psi, l$, and $h$ within this area, we can achieve the minimum values of reliability index $\beta_{\min }$, and safety factor $K_{s \min }$, and their corresponding $(\psi, l, h)$.

\subsection{Example Analysis}

Example (I) (see [15]). There is a slope whose height and slope angle are $4.5 \mathrm{~m}$ and $20^{\circ}$, respectively, with a sand bed beneath which helps to drain water away adequately. The natural unit weight of soil is $r=16 \mathrm{kNm}^{-3}$. The average efficacious shear strength and variation coefficient are $\mu_{c}^{\prime}=2.159 \mathrm{kPa}$, $\delta_{c}^{\prime}=30 \%, \mu_{\tan \varphi^{\prime}}=0.312$, and $\delta_{\tan \varphi^{\prime}}=2.5 \%$, respectively. Find out the failure probability of the given slid surface. The safety factor was calculated approximately to be $K_{s}=1.25$ with a vertical slice method in [15]. The failure probability was calculated to be $P_{f}=0.002(\beta=2.901)$ with statistics formulas.

With the novel method (Spoke model) proposed in this work, the safety factor is calculated to be $K_{s}=1.252$. If $c$ and $\tan \varphi^{\prime}$ are regarded as normal random variables, and they are not interrelated, the corresponding reliability index is calculated to be 2.883 with the Spoke model. 
TABle 2: Calculation Results for Example (III).

\begin{tabular}{|c|c|c|c|c|}
\hline & & \multicolumn{3}{|c|}{ Corresponding coordinates of the minimum value } \\
\hline & & $\psi\left({ }^{\circ}\right)$ & $l(\mathrm{~m})$ & $h(\mathrm{~m})$ \\
\hline \multirow{2}{*}{$\begin{array}{l}\text { Safety index (variation coefficients of } c \text { and } \tan \varphi \text { are } 0.2 \text { and } \\
0.275 \text {, resp.) }\end{array}$} & $K_{s \min }=1.38$ & 7.4 & 1.32 & 11.09 \\
\hline & $\beta_{\min }=1.60$ & 7.6 & 1.34 & 11.13 \\
\hline \multirow{2}{*}{ Safety index (variation coefficients of $c$ and $\tan \varphi$ are both 0.15 ) } & $K_{s \min }=1.38$ & 7.4 & 1.32 & 11.09 \\
\hline & $\beta_{\min }=2.37$ & 6.4 & 1.45 & 10.98 \\
\hline
\end{tabular}

Example (II) (see [8]). There is a homogeneous simple slope whose height $(h)$ is $50 \mathrm{~m}$, slope proportion $(m)$ is 3.25 , bulk density of soil $(r)$ is $19.62 \mathrm{kNm}^{-3}$, coefficient of friction is $\tan \varphi=0.2$, and adhesion stress $(c)$ is $58.66 \mathrm{kPa}$. Work out the location of the most dangerous slide arc and minimum value of safety factor.

The calculated results with the Spoke model proposed in this work and the corresponding results in [8] are listed in Table 1 .

Example (III) (see [7]). There is a clay slope whose height is $10.0 \mathrm{~m}$, and the proportion of slope is $1: 2$. The average value and standard deviation of $c$ and $\tan \varphi$ are $20 \mathrm{kPa}, 0.1817$ and $4.0 \mathrm{kPa}, 0.05$, respectively, and all of them are considered to be of normal distribution and not interrelated. The unit weight of soil is $16.0 \mathrm{kNm}^{-3}$, considered as a fixed value. Find out the minimum value of safety factor and reliability index of the slope.

With the Spoke model in this work, we take $\Delta \psi=0.2^{\circ}$, $\Delta l=0.05 \times H_{0} \sin \psi / 2 \sin \alpha_{0} \sin \left(\alpha_{0}-\psi\right)$, and $\Delta h=0.05 \times$ $\left[\left(H_{0} / \sin \alpha_{0}-(s-l) / \cos \left(\alpha_{0}-\psi\right)\right) / \tan \left(\alpha_{0}-\psi\right)-H_{0} / \cos \left(\alpha_{0}-\psi\right)\right]$ as the step length to search solution. The results are listed in Table 2.

According to the analysis of Examples (I) and (II), the calculated results with Spoke model and traditional slice methods are subequal. Example (III) indicates that minimum safety factor and minimum reliability index are not achieved on the same slip surface. In addition, the variability of soil parameters affects the minimum reliability index and the position of its corresponding slip surface but possesses little influence on the minimum safety factor and the position of its corresponding slip surface.

It is worth noting that the expression of safety factor has a clear physical meaning in this work. With the calculation program built, it is convenient for data input, and the calculation process is quick. With this method for reliability analysis of slopes, the limit state function can be achieved which directly results in the reliability index.

\section{Conclusion}

The explicit expression of safety factor in Spoke model proposed in this work requires no repeated iterative solution, as well as can be developed into a limit state function required by calculation of the reliability index. The Spoke model avoids complicated repeated iterative solution as required in traditional slice methods as well as eliminates the errors arising from oversimplified assumption.
According to the example analysis, the slip surface with minimum reliability index is not in coincidence with, but similar to, the one with minimum safety factor. We can find out the minimum safety factor and its corresponding coordinates first, then search the minimum reliability index nearby.

The minimum reliability index and location of its corresponding slip surface are related with soil parameters such as weight density " $G$," cohesion " $c$," and internal friction angle " $\varphi$." The minimum safety factor and location of its corresponding slip surface have no relation with variability of soil parameters.

This work only discusses the calculation analysis of reliability of simple slopes. The failure surface is supposed as a circular arc, and some simplifications are utilized on the resistance calculation of slip surface caused by the stress between slices. The influence of simplified treatment in this work on calculation results could be a direction of further research.

\section{References}

[1] W. Lian-ying and C. Hong-kai, "Genetic algorithm analysis on global stability of landslide and its application," Journal of Chongqing Jiaotong University, no. 5, pp. 907-910, 2009.

[2] A. Sengupta and A. Upadhyay, "Locating the critical failure surface in a slope stability analysis by genetic algorithm," Applied Soft Computing Journal, vol. 9, no. 1, pp. 387-392, 2009.

[3] Z. B. Peng, J. Li, and W. X. Peng, "Application analysis of slope reliability based on Bishop analytical method," Journal of Central South University, vol. 41, no. 2, pp. 668-672, 2010.

[4] Y. Cheng-Zhi, "Reliability analysis of slope stability using Janbu method," Mining Engineering, no. 1, pp. 15-17, 2008.

[5] B. K. Low and W. H. Tang, "Probabilistic slope analysis using Janbu's generalized procedure of slices," Computers and Geotechnics, vol. 21, no. 2, pp. 121-142, 1997.

[6] L. Liang and C. Xue-song, "Application of Rosenblueth method in reliability analysis of soil slopes," Advances in Science and Technology of Water Resources, no. 3, pp. 53-66, 2012.

[7] W. Ping and L. Dong-sheng, "Calculative research on reliability of simple soil slope," Rock and Soil Mechanics, vol. 22, no. 4, pp. 494-498, 2001.

[8] W. Ping and L. Dong-sheng, "Spoke model for evaluation of safety factor for soil slope," Engineering Mechanics, vol. 19, no. 3, pp. 148-152, 2002.

[9] Q. Quan, L. Dao-jin, and M. Gang, Theory and Applications Reliability Stochastic Finite Element Methods, Tsinghua University Press, Beijing, China, 2006. 
[10] G. Jin-xin, Computational Method for Reliability of Engineering Structure, Dalian University of Technology Press, Dalian, China, 2003.

[11] D. Wei and S. Rahman, "A multi-point univariate decomposition method for structural reliability analysis," International Journal of Pressure Vessels and Piping, vol. 87, no. 5, pp. 220-229, 2010.

[12] L. Yong, W. Ping, and L. Dong-sheng, "New algorithm for calculation of reliability indexes of structures," Chinese Journal of Rock Mechanics and Engineering, no. 5, pp. 736-739, 2002.

[13] K. Farah, M. Ltifi, and H. Hassis, "Reliability analysis of slope Stability using stochastic finite element method," Procedia Engineering, vol. 10, pp. 1402-1407, 2011.

[14] Q. Jia-huang and Y. Zong-ze, Geotechnical Principles and Computation, Water Conservancy and Electric Power Press, Beijing, China, 2003.

[15] Editor Committee of Handbook of Rock and Soil Engineering, Handbook of Rock and Soil Engineering, Architecture Industry Press, Beijing, China, 1994. 


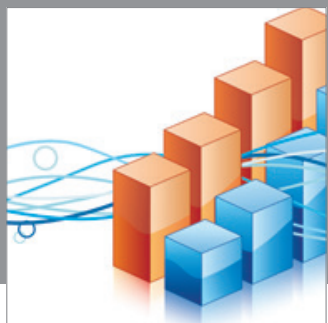

Advances in

Operations Research

mansans

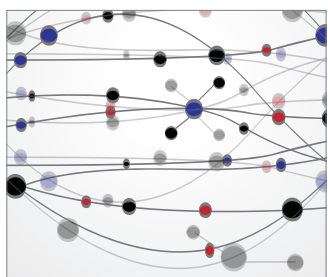

The Scientific World Journal
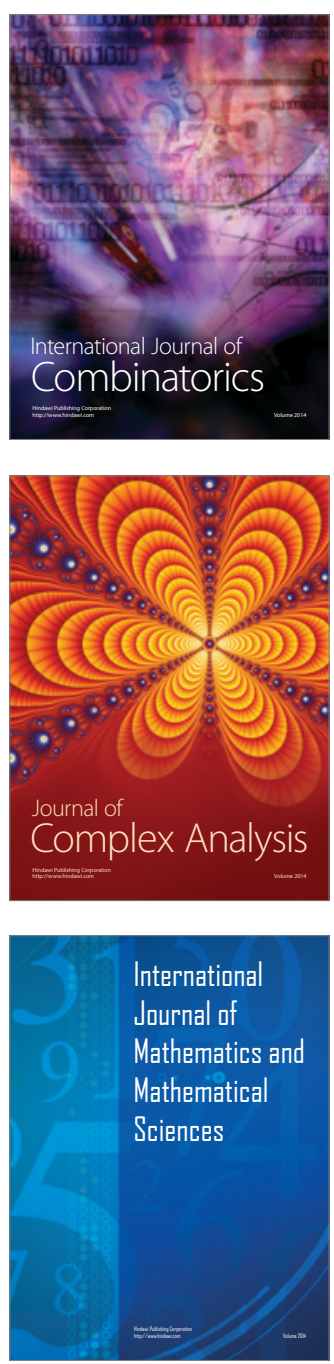
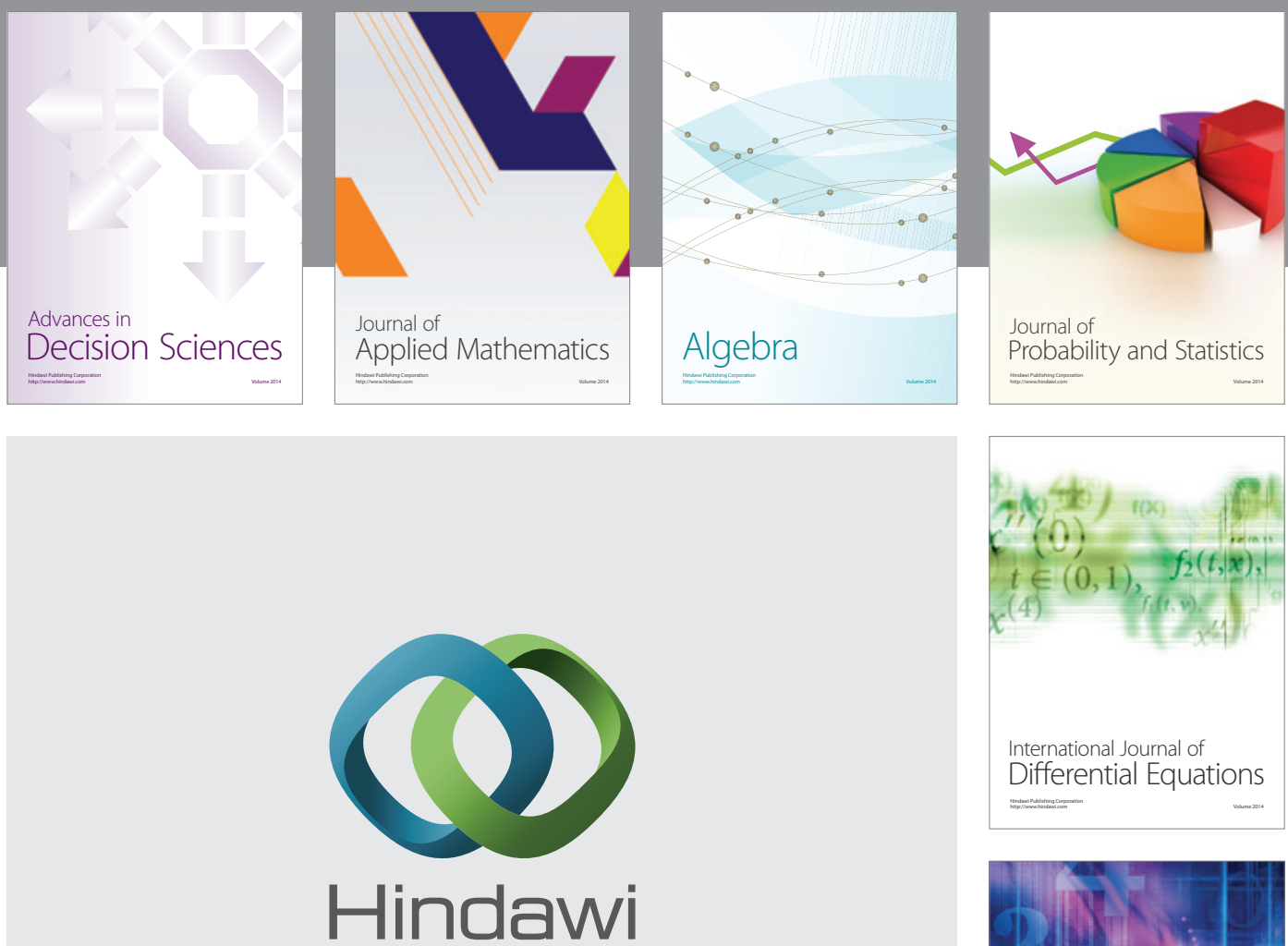

Submit your manuscripts at http://www.hindawi.com
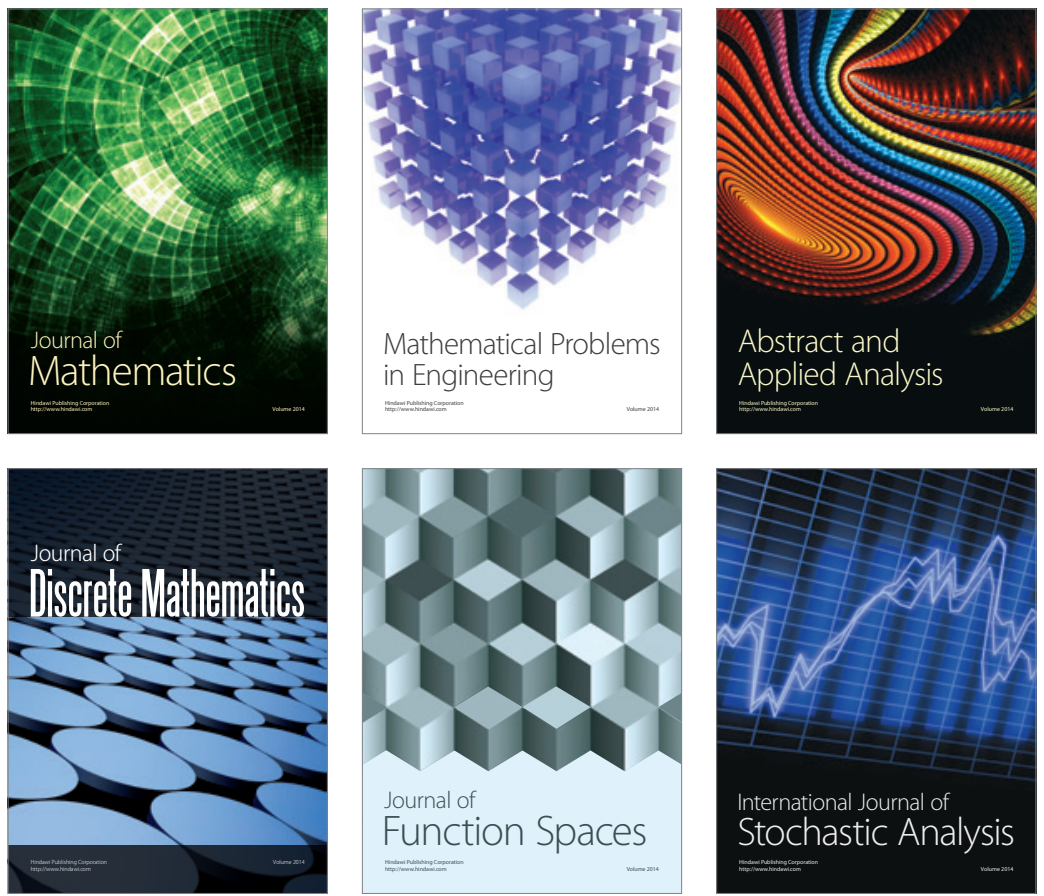

Journal of

Function Spaces

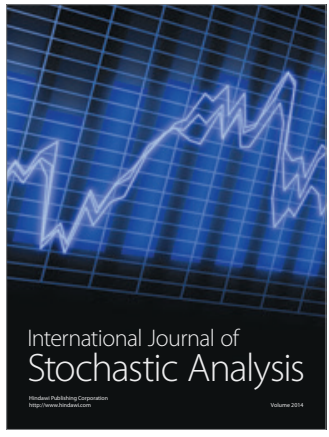

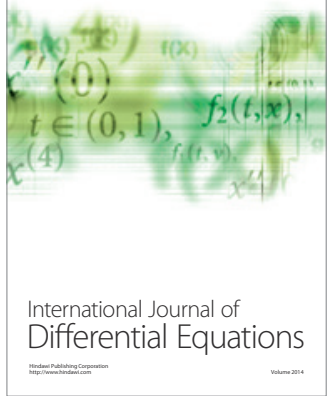
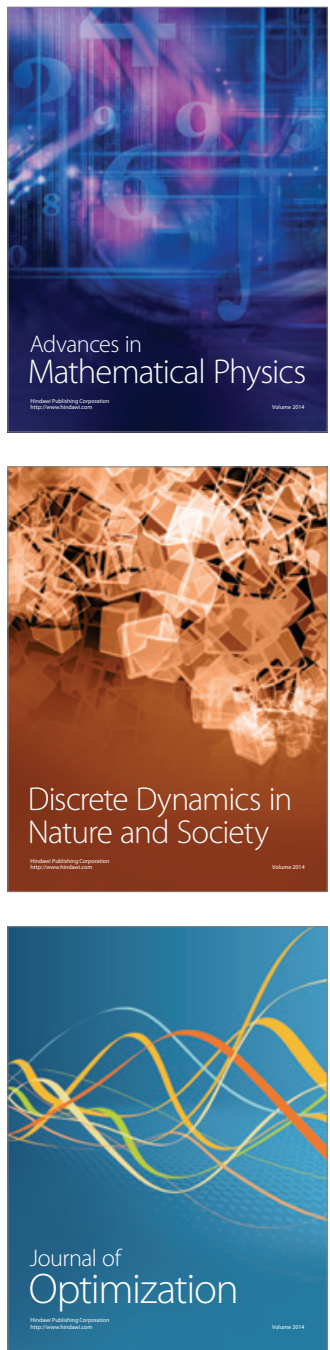